\title{
Exploration for matter stability \& the material basis and mechanism for the interaction of light, electricity and magnetic
}

\author{
- The physical world described with the basic gravitino theory
}

\section{Zhengrong Tong}

Basic Physics Research Department, Shanghai Development Research Institute, Development Research Center, The State Council of P.R. China, Shanghai, China; tzr01@yahoo.cn

Received 21 January 2012; revised 24 February 2012; accepted 6 March 2012

\begin{abstract}
This paper is about the theory of "basic gravitinoes" (in early papers, it was referred to WG) in the material world, which make up the "dark matter" and the elementary particles as well. The strong interaction is generated by the Pressure Effect, which is resulted in the accumulation of the overall gravitation from all the galaxy gravitinoes. The Model for Stable-state Particle Basic Body indicates three stable states which correspond to protons, electrons and neutrons respectively and have been proven by all the particle collision experiments. It's the root cause that only the short-lived particles resulted in the collisions. The study is supported by all experiments of light propagation, especially the waveparticle duality, and tries to make the theoretical interpretation for three laws of electromagnetic experiment so that the Maxwell's field equations are derived ultimately and theoretically.
\end{abstract}

Keywords: Basic Gravitino; WG Condensation; "Droplets" under the Strong WG Pressure; B Body; Wave-Particle Duality Standing Wave State

\section{BASIC GRAVITINO AND THE STRONG INTERACTION}

Basic gravitino* (in early papers, was referred to $\mathrm{WG}$ ) theory and its research findings related to the mechanism of the stable existence of matter show that protons, electrons, neutrons have the definite mass and definite amount of charges because they are all composed of the same basic gravitinoes [1-3]. Moreover, the strong interaction is not generated from inside the particles but by the Pressure Effect, which is resulted in the accumulation of the overall gravitation from all the galaxy gravitinoes. It has been discovered that there is a mathematic relation among the mass of universe, the mass of the basic gravitino and the strong interaction as follows: [4]

$$
F=-\frac{G m_{w{ }_{u}}}{r^{2}}\left(r \rightarrow r_{B}\right)
$$

According this model, the intensity of the strong interaction is $M_{u}$ times of the gravitational forces between particles $\left(M_{u}\right.$ is the total mass of the universe), which conforms to the actual condition for the intensity of strong interaction. Meanwhile, it indicates that it is a misunderstanding of considering that the strong interaction exists inside the particles.

\section{A DYNAMIC BALANCE EQUATION AND A MATHEMATICAL MODEL FOR THE STABLE STATE OF FUNDAMENTAL PARTICLES}

The stable particles are the "fog grains" condensed with the "basic gravitino" (WG) under the overall strong pressure of the basic gravitinos in the universe, which are similar to the "droplets" in the saturation of gas. This research gives a dynamic balance equation and a mathematical model for the stable state of fundamental particles: [5]

"Note: This theory proves that there is a kind of "basic gravitino" (i.e. WG) in the material world; and it not only makes up the "dark matter" in the universe but also the elementary particles that physics has studied and found.

Related research has found a method to determine the mass-energy value of the "basic gravitino" and given the theoretical value at $m_{w}=$ $3.636 \times 10^{-45} \mathrm{~kg}$, [12] which tallies with the experimental values from the detection for free particles in space that observed by "Double Star" [De Broglie] [13] and "binary pulsar" [Seal Berg] [14]. Please see Dark Matter Physics, Chapters 1, 2, and 4 by Tong Zhengrong, published by Zhuhai Publishing House in 2008 [15] (ISBN 978-7-54530001-7).

In November 1996, Tong Zhengrong delivered a keynote speech on gravitino theory at the national academic conference of Chinese Society of General Relativity and China Institute of Celestial Gravity. 


$$
I_{0}=I=A N d_{W}^{2}
$$

The study leads to the concept of "Stable-state Particle Basic Body", or "B Body", and proves the unique existence of "B body". The model gives directly the three stable states which correspond to protons, electrons, neutrons respectively. This conclusion has been proved by all the particle collision experiments and really involves the cause and mechanism of the stable existence of matter. Scientists have been using strong energy to accelerate particles, including the Large Hadron Collider (LHC) project by European Organization for Nuclear Research Centre (CERN), but they fail to get anything but shortlived particles in a flash only. They fail to get any new "stable particles" different from protons, electrons as we have known to form stable matter.

\section{THE PROPERTIES OF SPACE BASIC GRAVITINO}

The properties of space "dark matter", which the Basic gravitino constitutes can be summarized as follows:

- Gravity is the basic property of the basic gravitino but the direct gravitational force between particles is too weak to aggregate to larger particles. WG falls within the scope of elastic particles. Its basic equations are as follows: [6]

$$
\frac{\partial F_{\mu \nu}}{\partial X_{v}}-\mu^{2} A_{\mu}=0 ; \frac{\partial F_{\mu v}}{\partial X_{\lambda}}+\frac{\partial F_{v \lambda}}{\partial X_{\mu}}+\frac{\partial F_{\lambda \mu}}{\partial X_{v}}=0
$$

A team of Chinese authoritative domestic scholars once conducted a mathematical analysis on the theory for basic gravitino constituting the entire universe. They came to conclusion that WG theory can really solve the problem of dark matter in the universe (see "Dark Matter Physics", Chapter 11.1. WG star, the mathematical analysis for WG constitutes the entire universe).

- The gravitational accumulation arising from the "basic gravitino" (WG) all over the universe results in the WG pressure in space. Calculation shows that WG pressure is consistent with the intensity of the strong interaction and also shows that the elementary particles are the "droplets" under the strong WG pressure in entire universe, similar to the droplets in the saturated gas. There are stable-state solutions in Mathematical models corresponding to the proton, electron the neutron.

- The average speed of the "basic gravitino" (WG) is at the same magnitude as the speed of light.

- The "basic gravitino" (WG) in the universe has the extremely small mass density - at the magnitude of $10^{-28} \mathrm{G} / \mathrm{cm}^{3}$. The media matter plays a very small role in the non-high-speed state of moving objects; however, it has the extremely high amount density - the surface of a sphere with the diameter of 1 Angstrom is hit by the WGs in the space at the magnitude of $10^{8-14}$ times per second.

- WG has much stronger permeability than a neutrino.

The five unique properties mentioned above provide the material conditions and essential mechanism for researching the light and propagation with light speed in its wave-particle duality standing wave state. Meanwhile it lays the foundation for the action mechanism of the electromagnetic field and the theoretical interpretation of three laws of electromagnetic experiment, as well as the inference of Maxwell's field equations.

\section{THE WAVE-PARTICLE INTERFERENCE PHENOMENON OF LIGHT}

The researches have shown that the motion of light is a kind of wave-particle interference phenomenon between the WG particle pulses and WGs in the space as the media. The mechanism is described as follows: [7,8].

Assuming that the light source stimulates some WG pulses under certain action, the media (matters) will fluctuate inevitably when the beam of particles injects into the media which composed with the same WGs. The fluctuation frequency is subject to the beam source, the energy of beam and other factors. The media matters fluctuations in turn counteract on light sources inevitably, thus forcing the light sources to stimulate the WGs with a forced vibration frequency. We have sophisticated mathematical wave theory to deal with the physical problems. This is obviously a typical standing wave problem.

The superimposition of the WG pulse stimulated by the light source with certain frequency and the fluctuation from the media matters results in the standing wave form of interference. This motion property determines some special physical phenomena. For example, the energy of the light is intermittent; it possesses the physical characteristics of particles, as well as all the wave characteristics, i.e. light has impulse; light has light pressure and the other properties. In fact, the wave particle duality model of light propagation can be simulated in the following experiment.

A specially-designed high-frequency molecular beam shot "gun" injects the air molecules with the "ultrasonic" frequency into the air-filled space. All the wave-particle duality features can be observed in this experiment; we can check its linear spreading, radial "wave pressure" (equivalent to light pressure), interference and diffraction... The model also reveals that the wave-particle duality will be quite obvious as long as the mass-energy difference between the radiation beam particle flow of the source and "WG" falls in certain range. The beam of heavy particles with much greater particle mass-energy than the WGs mainly renders the character of particles, which conforms to the facts. This model also gives a 
rational explanation to the polarization of light, photoelectric effect and other experiments ("Dark Matter Physics", Chapter 5 \& 18).

\section{THE MECHANISM OF THE ELECTROMAGNETIC INTERACTION}

Basic gravitino theory makes a thorough re-search of electrostatic interactions. It has found that the outbound radiation frequency of the "Particle Basic Body" (B body) should be divided into two major parts, one is the vibration frequency of the "B body" as the core of mass $\left(v_{B}\right)$, and the other, the radiation frequency of the WG cloud outside the core of the "B body" $\left(v_{w}\right)$. Obviously the vibration frequency from the core of mass is much lower than the radiation frequency of orbital WGs. They have completely different spectrums, and $v_{w} \gg v_{B}$. With regard to the proton body and the electron body only, the proton body's outbound radiation is mainly the $v_{w}$, called the high frequency body; while the electron body's outbound radiation is mainly $v_{B}$, called the low frequency body. Electric interaction is attributed to the dynamic effect under the resonate principle between the bodies with different frequencies and between the bodies with the same frequencies. In the nature, the quantity of electricity is defined as the flux of the outbound external radiation WGs from the charged objects. So the electric charge quantization essences, such as "Coulomb's law", "Gauss Law", "Biot-Sha Cutting Law" could be naturally derives from the premise above [9].

In regard to magnetism, a conclusion drawn from this theory is that the magnetism is the WG absorption or release field and the complex field for the spatial WG whirlpool. The related experimental laws also could be derived from the theory [10].

\section{ABOUT THE SYMPOSIUM}

The study also conducts a symposium on the following issues:

- The material basis, mechanism interpretation and mathematical derivation associated with the introduction of Plural form of the quantum mechanical wave function [11];

- The light standing wave state of wave-particle interference under gravitino theory; The following-up principle about media system of the light propagation;

- The paradox - the atomic system is stable, but its electron orbital probability movement causing inevitable and ceaseless electromagnetism energy radiation;

- Light propagation and the "Doppler effect" related to the propagation distance...

Here is not described in details one by one.

\section{REFERENCES}

[1] Tong, Z.R. (2002) The WG theory, 4.1-4.2. http://tongzr.w1.hostlogin.cn/Ewgrrt/chapter4.htm

[2] Tong, Z.R. (2002) The discovery of dark matter, 1.2-1.4. http://tongzr.w1.hostlogin.cn/Ewgrrt/chapter1.htm

[3] Tong, Z.R. (2002) The math-physical model for the universe consisting of dark matter WG. http://tongzr.w1.hostlogin.cn/Ewgrrt/chapter11.htm

[4] Tong, Z.R. (2002) Strong interaction. http://tongzr.w1.hostlogin.cn/Ewgrrt/chapter7.htm

[5] Tong, Z.R. (2002) A math-physical model of stable elemental particle formation- "Basic body of particles", 8.1-8.5. http://tongzr.w1.hostlogin.cn/Ewgrrt/chapter8.htm

[6] Tong, Z.R. (2002) The equation of motion for the WG original particle. http://tongzr.w1.hostlogin.cn/Ewgrrt/chapter10.htm

[7] Tong, Z.R. (2002) The medium of light propagation, 2.1-2.2. http://tongzr.w1.hostlogin.cn/Ewgrrt/chapter2.htm

[8] Tong, Z.R. (2002) The dark matter and the nature of light. http://tongzr.w1.hostlogin.cn/Ewgrrt/chapter5.htm

[9] Tong, Z.R. (2002) The essence of electric interaction, 12.1-12.5. http://tongzr.w1.hostlogin.cn/Ewgrrt/chapter12.htm

[10] Tong, Z.R. (2002) The vortex field of dark matter and magnetic field. http://tongzr.w1.hostlogin.cn/Ewgrrt/chapter13.htm

[11] Tong, Z.R. (2002) The medium of light propagation and the complex wave function in quantum mechanics. http://tongzr.w1.hostlogin.cn/Ewgrrt/chapter15.htm

[12] Tong, Z.R. (2002) The mass of the dark matter wg and two experimental data, 9.1-9.2. http://tongzr.w1.hostlogin.cn/Ewgrrt/chapter9.htm

[13] De Broglie, L. (1940) La mechanique ondulatoire du photon, une Nouvelle theorie de la lumiere, Volcanol, Hermann, Paris, 39-40.

[14] G. Feinberg (1969) Binary pulsar. Nature, 221, 525.

[15] Tong, Z.R. (2006) Dark matter physics. Zhuhai Publishing House, Zhuhai. 